digkeit $v$ ebener Wellen mit der Frequenz $v$ $v=\sqrt{\frac{k T_{\mathrm{e}} \nu^{2}}{m\left(v^{2}-v_{0}^{2}\right)}}$, wobei $v_{0}$ der schon in $\mathrm{Ab}-$ schn. 1 genannten Elektronenkonzentration gemäß $v_{0}^{2}=\frac{n_{0} e^{2}}{\pi m}$ entspricht; $T_{\mathrm{e}}$ ist die Temperatur des Elektronengases. Für Werte von $v_{0}$, die sich nur noch wenig von $v$ (in unserem Falle $=$ Senderfrequenz) unterscheiden, nimmt $v$ rasch extreme Werte an. $v_{0} \rightarrow \boldsymbol{v}$ bedeutet nun, daß sich die Elektronen schließlich in gleicher Phase bewegen. Dies könnte in unserem Falle eine Erklärung für die gemäß den obigen experimentellen Befunden bereits vermuteten starken Ringströme darstellen. Die Lindersche Theorie müßte dann allerdings noch für den Fall des hier benutzten Rotationsfeldes ausgebaut und hinsichtlich des Einflusses eines überlagerten statischen Magnetfeldes ergänzt werden. Die Durchrechnung dieser Aufgabe stößt aber auf beträchtliche mathematische Schwierigkeiten.

Schließlich kann man noch aus der schon mehrfach genannten Formel $v_{0}^{2}=\frac{n_{0} e^{2}}{\pi m}$ die zur Resonanz notwendige Elektronenkonzentration errech- nen. Für eine mittlere Frequenz von $19 \mathrm{MHz}$ wird $n_{0}=4,4 \cdot 10^{6} / \mathrm{cm}^{3}$.

* Anm. b. d. Korr.: Der experimentelle Befund der starken Abhängigkeit der magnetischen Wechselfeldstärke in der Gasentladung von Anodenspannung und statischem Magnetfeld legte in erster Linie die Deutung des gefundenen Effektes als eine von der Elektronenkonzentration stark abhängige Plasmaschwingung nahe. Es besteht jedoch offensichtlich eine Analogie hinsichtlich Versuchsanordnung (statisches Magnetfeld senkrecht zum magnetischen Wechselfeld) und Phänomenologie (Resonanz in der Leistungsaufnahme) mit Versuchen der Bestimmung der ferround paramagnetischen Erscheinungen fester oder flüssiger Körper bei hohen Frequenzen ${ }^{7}$. Es ist daher durchaus möglich, daß die beschriebenen Erscheinungen ähnlich wie diese Versuche erklärt werden können. Es sei dabei noch darauf hingewiesen, daß die Versuchsanordnung zunächst zum Zwecke der Steigerung der Leistungsfähigkeit der Hochfrequenzionenquelle getroffen wurde und nicht speziell zur Klärung der Frage des Auftretens von Plasmaschwingungen oder von Resonanzerscheinungen bei in Gasentladungen induzierten magnetischen Momenten hinsichtlich eines statischen äußeren magnetischęn Feldes. Es ist durchaus möglich, daß beide Effekte unter gegenseitiger Beeinflussung auftreten.

7 J. H. E. G r if f ith s, Nature [London] 158, 670 [1946]; H. C. Torrey, E. M. P u r cell u. R. V. P o und, Physic. Rev. 69, 680 [1946]; Ch. Kittel, ebenda 73, 155 [1948]; F. B l o ch, W. W. Han s en u. M. P a c k a rd, ebenda 70, $474[1946]$.

\title{
Untersuchungen an Funkenschallwellen mit Hilfe von Röntgenblitzen Von Werner Schaaffs
}

Aus dem Physik.-techn. Laboratorium des WWM der Siemens \& Halske AG

(Z. Naturforschg. 4 a, 463-472 [1949]; eingegangen am 1. November 1948)

Durchleuchtet man mit Hilfe von Röntgenblitzen einen elektrischen Funken in einer dielektrischen Flüssigkeit, z. B. Trichloräthylen, im Augenblick des' Durchschlags, so nimmt man nur einen feinen verschwommenen Funkenkanal ohne Ausstrahlung von Schallenergie wahr. Erst einige Zeit später beginnt sich der Durchschlag deutlich in einen zentralen Entladungsraum und einen ihn ringförmig umgebenden Verdichtungsraum aufzuspalten. Dann läuft in etwa $10^{-6} \mathrm{sec}$ explosionsartig eine Schallwelle mit etwa $10 \mathrm{~km} / \mathrm{sec}$ Geschwindigkeit unter dynamischen Drucken zwischen $10^{4}$ und $10^{5} \mathrm{kp} / \mathrm{cm}^{2}$ ab, die aber rasch wieder an Geschwindigkeit verliert. Der sich dabei ausdehnende Entladungsraum sendet fortwährend in die schon vorverdichtete Umgebung schnelle Schallwellen aus, die den Verdichtungsraum erfüllen und die charakteristische Ausprägung der Funkenschallbilder unter mittleren Dichtesteigerungen um + 30\% und unter dynamischen Drucken um $10^{4} \mathrm{kp} / \mathrm{cm}^{2}$ verursachen. Nach etwa 50 Mikrosekunden ist die Schallgeschwindigkeit wieder auf ihren konstanten Normalwert von $1050 \mathrm{~m} / \mathrm{sec}$ abgefallen.

Die Funkenschallbilder sind nicht nur von dem Zeitmoment ihrer Aufnahme und der jeweiligen Entladungsstromstärke, sondern auch von der Dicke der Flüssigkeitsschicht, in der die Schallwellen laufen, abhängig. Die Ausbreitungsgeschwindigkeit des Verdichtungsraumes nimmt mit abnehmender Schichtdicke schneller als die Ausdehnungsgeschwindigkeit des zugehörigen Entladungsraumes ab. Dadurch kommt in sehr dünnen Schichten eine außerordentlich starke Kompression der Materie zustande, die sich, wie an verschiedenen Flüssigkeiten gezeigt wird, in einer Verdoppelung und Verdreifachung der Dichte und damit einer beträchtlich erhöhten Röntgenstrahlabsorption bemerkbar macht. Der Verdichtung ist aber dadurch eine Grenze gesetzt, daß mit abnehmendem Elektrodenabstand der Durchschlag nicht mehr durch einen Hauptkanal, sondern durch viele zusätzliche kleine Funkenkanäle vor sich geht. 
$\mathrm{W}$

enn man einen Funkendurchschlag durch ein flüssiges Dielektrikum mit Hilfe eines Röntgenblitzes parallel zur Funkenbahn durchleuchtet, so beobachtet man einen zentralen Gasentladungsraum E, der von einem Verdichtungsraum D ringförmig umgeben wird ${ }^{1}$. Im Verdichtungs * raum, der sich mit Überschallgeschwindigkeit ausbreitet, erfährt die Materie eine so starke Kompression, daß die damit verbundene Schwächung der Röntgenstrahlung die photographische Aufnahme und Auswertung der Erscheinung ermöglicht (Abb.1). Diese Erscheinung soll im Hinblick auf folgende spezielle Fragestellungen näher behandelt werden:

1. Wie sieht der Entladungsraum im Augenblick des elektrischen Durchschlags, also nach etwa $10^{-7} \mathrm{sec}$, aus?

2. Wie verändert sich in einem gegebenen Zeitmoment nach dem Durchschlag das Erscheinungsbild des Effekts bei Veränderung des Elektrodenabstandes?

3. Mit welcher Geschwindigkeit laufen bei gegebenem Elektrodenabstand die Funkenschallwellen ${ }^{2}$ $a b$ ? Besteht für bestimmte Zeitspannen nach dem Durchschlag eine starke Dispersion der Schallgeschwindigkeit?

4. Welche Verdichtungen der Materie können bei gegebener Entladungsstärke erzielt werden, und wie groß sind die zugehörigen dynamischen Drucke?

5. Welche Fehlerquellen müssen bei der Auswertung der Aufnahmen berücksichtigt werden?

\section{Experimentelles}

1. Apparat zur Messung des Entladungsraumes im Augenblick des Durchschlags

Um den elektrischen Durchschlag im ersten Moment sichtbar zu machen, muß die Zeitverzögerung zwischen elektrischem Durchschlag und Röntgenblitz von der Größenordnung der Dauer des letzteren sein, also etwa $10^{-7}$ sec betragen. Abb. 2 zeigt das Schema der Versuchsanordnung. In dem 2-poligen, dauernd evakuierten Röntgenblitzrohr RB steht der kegelförmigen, von dem Isolator I getragenen Wolframanode $\mathrm{A}$ eine plattenförmige Stahlkathode $\mathrm{K}$ gegenüber. Die Röntgenstrahlung tritt durch ein dünnes

1 W. Schagefs u. F. Trende e enburg, Z. Naturforschg. 3 a, 656-668 [1948]. Im folgenden stets mit ,.S. u. T." zitiert.

${ }^{2}$ Wir gebrauchen das Wort „Funkenschallwellen“ an Stelle von ,Knallwellen“, um der Eigenart ihrer Entstehung Rechnung zu tragen.
Aluminiumfenster ö aus, durchsetzt in einiger Entfernung die zwischen den beiden $2 \mathrm{~mm}$ starken gewölbten Aluminiumelektroden $e_{1}$ und $e_{2}$ (Krümmung:radius $130 \mathrm{~mm}$ ) befindliche Flüssigkeitsschicht aus Trichloräthylen und gelangt dann auf die photographische Platte P. Versuchsflüssigkeit, Blitzröhre und Kugelfunkenstrecke F sind in Reihe geschaltet. Der Kondensator C wird über einen Vorwiderstand auf Hochspannung aufgeladen, bis die Anordnung nach Naßgabe der Einstellung von $\mathrm{F}$ durch Überschlag zündet und sich der Kondensator momentan entlädt.

\section{Das Versuchsgefäß zur Erzeugung von Funkenschallbildern}

Die Röntgenblitzrohranlage zur Durchleuchtung von Funkenschallwellen in einem einstellbaren Zeitmoment ist in S.u.T. (Abschn. II) beschrieben worden. Da die Ausdehnung der Funkenschallbilder stark vom jeweiligen Elektrodenabstand bzw. von der örtlichen Dicke der Flüssigkeitsschicht abhängt, wurde das Versuchsgefäß, mit dem die Messungen vorgenommen wurden, verbessert; Abb. 3 zeigt einen Querschnitt. I ist ein Isolierkörper aus Igelit; T ein eiserner Hohlkörper, der durch drei gleichmäßig über den Umfang verteilte Schrauben $\mathrm{s}$ in der Höhe verstellbar ist und die obere, in einem mittleren Bereich flache Aluminiumelektrode $e_{2}$ trägt. Die untere Elektrode $e_{1}$ kann gewölbt sein, um den elektrischen Durchschlag möglichst in der Mitte der Versuchsflüssigkeit zu erzwingen. Wenn aber flache, feste Körper zur Untersuchung in die Flüssigkeit gebracht werden, so erweist sich eine ebene Elektrode $e_{1}$ als zweckmäßiger. Legt man großen Wert auf eine Steigerung der Bildkontraste, so kann man die Elektroden aus gewölbten Pertinaxplatten, die mit dünnem Aluminiumblech überzogen werden, anfertigen. Der Ort des elektrischen Durchschlags läßt sich meist nicht festlegen und ist nicht mit dem kleinsten Elektrodenabstand identisch. Die Folge ist, daß die Funkenschallbilder meist nicht genau kreissymmetrisch ausgebildet sind. Diese Unsymmetrie muß bei ihrer Auswertung, z. B. bei der Berechnung der Verdichtungen, beachtet werden.

\section{Kurzzeitmessungen mit der Röntgenblitzröhre}

Zur Bestimmung der Ausbreitungsgeschwindigkeit der Funkenschallwellen sind Kurzzeitmessungen erforderlich. Wie die Auslösung eines Röntgenblitzes durch den zu untersuchenden Vorgang gesteuert wird, ist in S. u. T. beschrieben worden. Für die gesamte Zeitverzögerung zwischen dem Funkendurchschlag im Versuchsgefäß und dem ihn durchleuchtenden Röntgenblitz sind die Zeitkonstante des Stromtorkreises und die Teilverzögerungen an der einstellbaren Verzögerungsfunkenstrecke und an der Zündelektrode der Blitzröhre maßgebend. Die letztere ist für die Erfassung jener kleinen Zeitmomente, in denen die Funkenschallwellen ihre größte Geschwindigkeit haben, von größter Wichtigkeit; sie hängt von der 

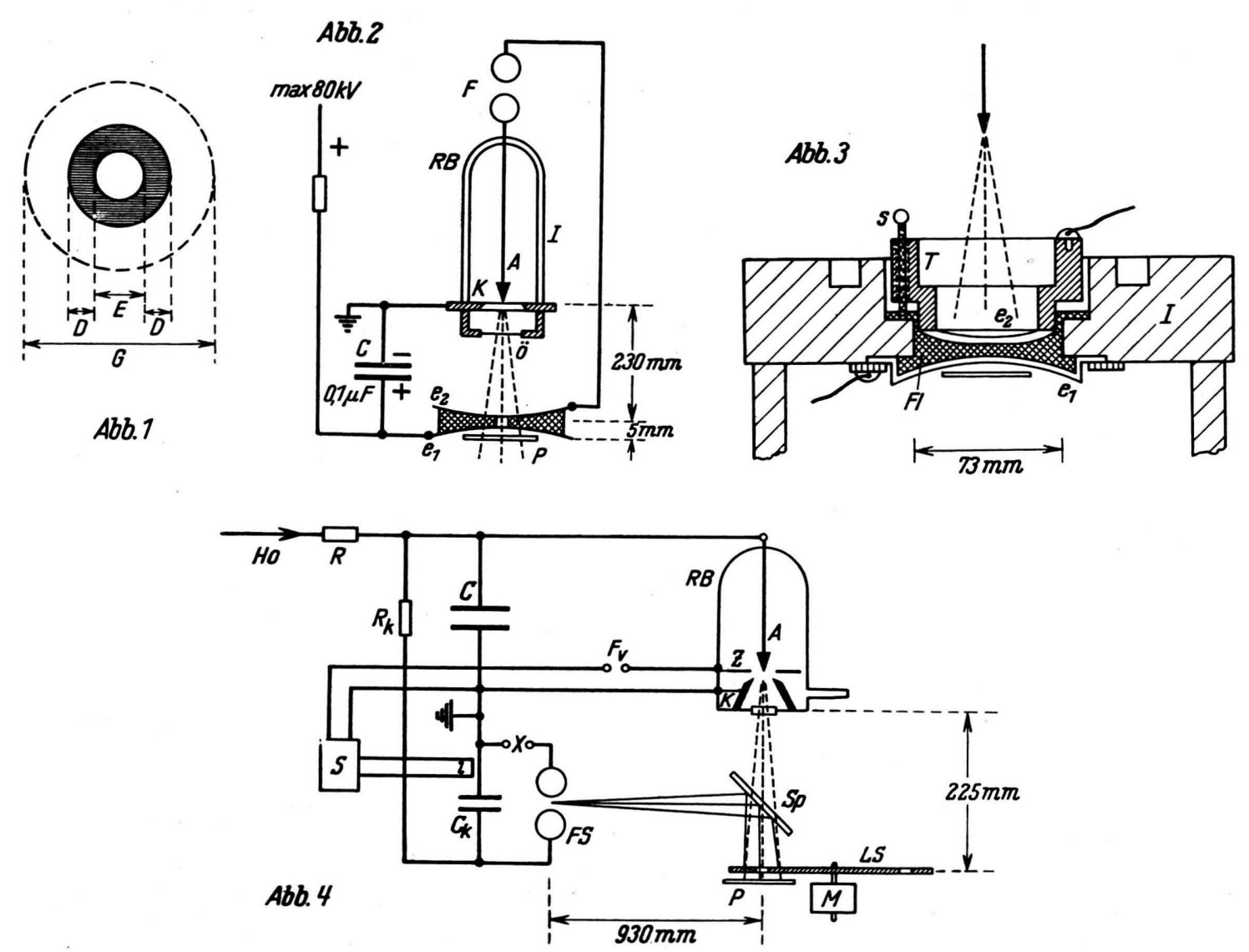

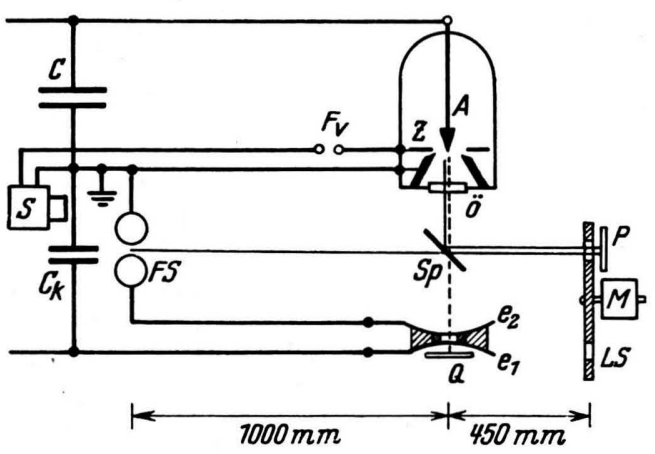

Abb. 5

Abb. 1. Schema eines Funkenschallbildes. Der Entladungsraum E wird vom Verdichtungsraum D ringförmig umgeben. $\mathrm{G}$ ist der von den Röntgenblitzen durchleuchtete Bereich.

Abb. 2. Schema der Versuchsanordnung zur Röntgendurchleuchtung von Funkenstrecken im Augenblick des elektrischen Durchschlags.

Abb. 3. Querschnitt durch das Versuchsgefäß zur Erzeugung von Funkenschallbildern.

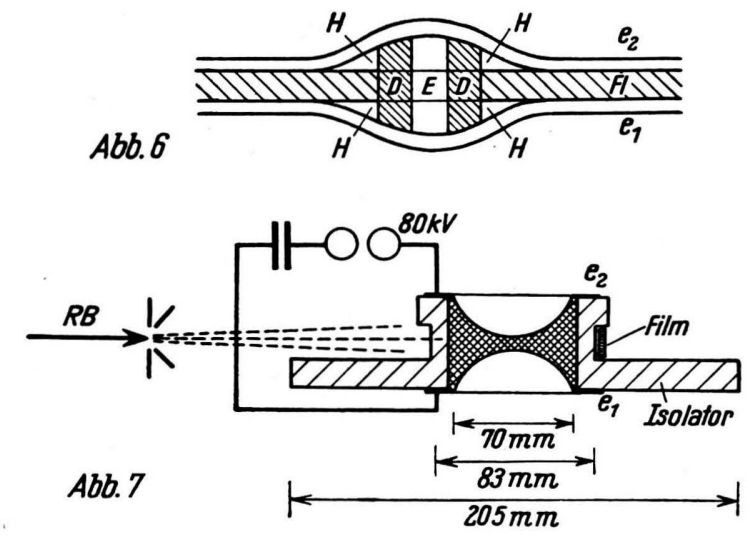

Abb. 4. Messung der Abhängigkeit der Zeitverzögerung $t$ von der Verzögerungsfunkenstrecke $\mathrm{F}_{\mathrm{v}}$ in der Röntgenblitz-Meßapparatur.

Abb. 5. Gleichzeitige Photographie eines Funkenschallbildes auf der Platte Q mit Röntgenblitz und der zugehörigen Zeit $t$ auf der Platte $\mathrm{P}$ mit 2 Lichtblitzen.

Abb. 6. Schema zum Problem der möglichen Deformation der Elektroden unter dem Einfluß desEntladungsdruckes.

Abb. 7. Versuchsanordnung zur Durchleuchtung des Elektrodensystems und des Funkens senkrecht zur Funkenbahn. 

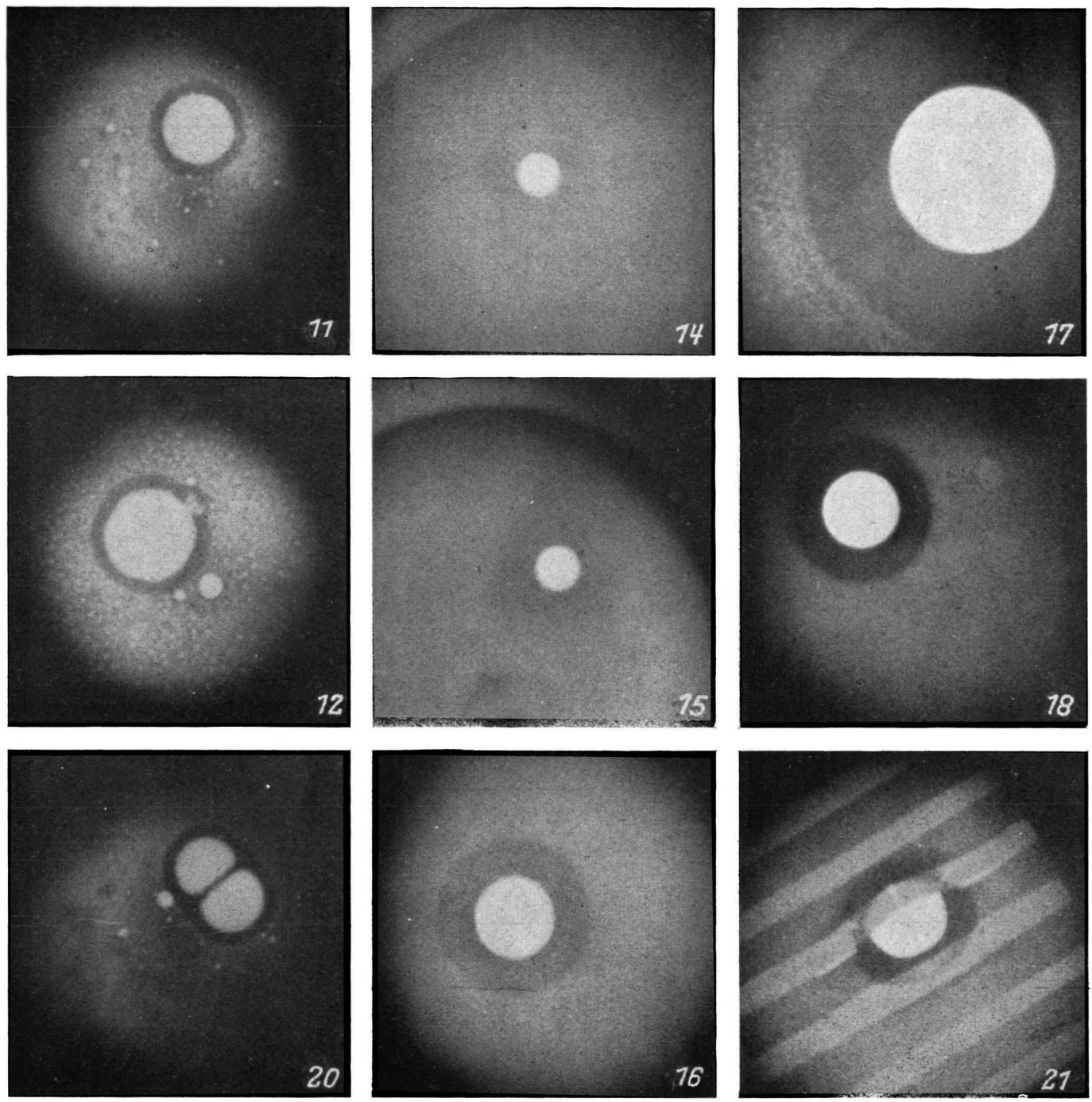

Abb. 11. Überschlag in Brombenzol bei $d=0,2 \mathrm{~mm}$ und

$$
t=21 \cdot 10^{-6} \mathrm{sec} \text {. }
$$

Abb. 12. Überschlag in Brombenzol bei $d=0,2 \mathrm{~mm}$ und $t=30 \cdot 10^{-6}$ sec.

Abb. 14. Schallemission im Zeitabschnitt $t_{3}$. Trichloräthylen. Bild zu den Kurven der Abb. 13.

Abb. 15. Schallemission im Zeitabschnitt $t_{3}$. Trichloräthylen. Bild zu den Kurven der Abb. 13.
Abb. 16. Schallemission im Zeitabschnitt $t_{4}$. Trichloräthylen. Bild zu den Kurven der Abb. 13.

Abb. 17. Schallemission im Zeitabschnitt $t_{4}$. Trichloräthylen. Bild zu den Kurven der Abb. 13.

Abb. 18. Ċberschlag in Trichloräthylen bei $d=0,6 \mathrm{~mm}$ und $t=25 \cdot 10^{-6}$ sec. Geschichtete Elektroden.

Abb. 20. Zusammenstoß zweier Funkenschallwellen in Äthylenbromid bei $d=0,4 \mathrm{~mm}$.

Abb. 21. Uberschlag in 'Trichloräthylen zwischen paral-

lelen Igelitstreifen von $1,6 \mathrm{~mm}$ Dicke hei $t=24 \cdot 10^{-6} \mathrm{sec}$. 
Kleinheit des Abstandes zwischen Zündelektrode und Kathode ab. Der höchstzulässige Elektrodenabstand ist $0,1 \mathrm{~mm}$. Es ist zwar wünschenswert, ihn noch wesentlich kleiner zu machen, doch tritt spätestens bei $0,05 \mathrm{~mm}$, vermutlich durch Ausbildung eines Lichtbogens, eine Brückenbildung zwischen Zündelektrode und Kathode ein, die die Röntgenblitzröhre unbrauchbar macht.

Die Bestimmung der Kurve des Zeitverzugs zwischen dem Durchschlag und dem dadurch ausgelösten Röntgenblitz in Abhängigkeit von der Verzögerungsfunkenstrecke $F_{\mathbf{v}}$ wird in dieser Arbeit mit Hilfe der Schaltung Abb. 4 vorgenommen. Im Strahlengang der Röntgenblitzröhre RB befindet sich ein schräggestellter Spiegel Sp, eine von einem Motor M betriebene Lochscheibe LS, und dicht unter dieser die photographische Platte P. Wird durch Annäherung der Kugeln der Kondensator $C_{k}$ über die Schaltfunkenstrecke FS entladen, so fixiert der von ihr ausgehende Lichtblitz nach Reflexion an Sp eine Stellung der Lochscheibe LS auf der Platte P. Der über das Kabel 1, die Stromtorzündschaltung $S$, die Verzögerungsfunkenstrecke $F_{v}$ an die Zündelektrode Z-des' Blitzrohres fortgeleitete Spannungsstoß bringt den Kondensator C über RB zur Entladung. Der von der Anode A ausgehende Röntgenblitz fixiert die rotierende Lochscheibe dann in einer anderen Lage. Aus der Tourenzahl des Motors und dem Wegunterschied der beiden Lagen wird der Zeitunterschied zwischen dem Durchschlag bei FS bzw. X und der Durchstrahlung mit dem Röntgenblitz berechnet. Bei $\mathrm{X}$ ist das Elektrodensystem mit der Versuchsflüssigkeit angeschlossen. Das Licht des Durchschlàgs, der die Funkenschallwellen erzeugt, kann nach Sachlage der Dinge zur Zeitmarkierung nicht benützt werden, doch kann`angenommen werden, daß dieser Durchschlag mit dem Zeitmoment maximaler Lichtaussendung der Schaltfunkenstrecke FS praktisch zusammenfällt.

Wünscht man, jedem Funkenschallbild die zugehörige Zeitverzögerung sofort zuzuordnen, so kann man die Versuchsanordnung nach Abb. 5 anwenden. Hier ist die Aluminiumscheibe, die bislang als Strahlenaustrittsöffnung des Blitzrohres gedient hat, durch ein Glasfenster ö ersetzt worden. Beim Funkendurehschlag im Elektrodensystem $e_{1} e_{2}$ wird gleichzeitig die Schaltfunkenstrecke FS durchschlagen, und der von ihr ausgesandte Lichtblitz markiert eine Stellung der rotierenden Lochscheibe auf der Photoplatte P. Eine gewisse Zeit später wird die Blitzröhre gezündet. Der von der Anode A ausgehende Röntgenblitz fixiert auf der Platte Q das Funkenschallbild, und der gleichzeitig damit von A ausgehende Lichtblitz markiert nach Reflexion am Spiegel Sp, der nur das halbe Strahlenfeld ausfüllt, auf der Platte $\mathrm{P}$ die zweite Stellung der Lochscheibe LS. Dabei wird stillschweigend angenommen, daß im Rahmen dieser Versuche die Einsätze von Röntgenblitz und Lichtblitz als gleichzeitig angesehen werden können.

Die Funkenschallbilder werden in dieser Arbeit durch oszillatorische Funken erzeugt. Demgemäß erscheinen die durch die Schaltfunkenstrecke FS auf der Platte $\mathrm{P}$ erzeugten Bilder der Lochscheibe LS mit einem abklingenden Band, welches anzeigt, daß die Entladungen in etwa 10 sec abgeklungen sind. Die. durch kurze und starke Kabel gehenden gedämpften Entladungen haben eine Frequenz von $v \approx 600 \mathrm{kHz}$ und eine maximale Stromstärke von $i \approx 850 \mathrm{Amp}$.

\section{Fehlerquellen und A f nahme- t e ch n ik}

Der Effekt der Sichtbarmachung von Schallwellen mit Röntgenstrahlen beruht lediglich auf der Ausnutzung von Dichteunterschieden in der durchstrahlten Materie. Die polychromatische Röntgenstrahlung des Röntgenblitzes' wird dabei nicht vorgefiltert, um ihre für den Kontrast so wertvollen weichen Komponenten nicht unwirksam zu machen. Das bringt allerdings den Nachteil mit sich, daß eine Dichtebestimmung des Verdichtungsraumes der Funkenschallbilder nicht auf Grund photometrischer Schwärzungsvergleiche vorgenommen werden kann. Die übliche Darstellung der Abhängigkeit des Schwächungskoeffizienten der Röntgenstrahlung von Wellenlänge, Ordnungszahl und Schichtdicke setzt nämlich monochromatische Strahlung und damit Konstanz des Schwächungskoeffizienten voraus, während in unserem Falle der kleinen Schichtdicken die Strahlenqualität noch durchaus veränderlich ist und der Schwächungskoeffizient mit zunehmender Schichtdicke noch abnimmt.

Was die Bildschärfe der Röntgenbilder betrifft, so setzt sie sich aus drei Anteilen zusammen, nämlich der durch die Breite des wirksamen Fokus hervorgerufenen Randunschärfe der Röntgenbilder, dann der durch die endliche Höhe des' Funkenschallraumes zwischen den Elektroden bedingten Höhenunschärfe, und schließlich der Bewegungsunschärfe, die dadurch entstehen kann, daß die Röntgenblitzdauer nicht mehr kurz genug ist, um einen sich beispielsweise mit $10 \mathrm{~km} / \mathrm{sec}$ ausbreitenden Vorgang exakt zu erfassen. Die Versuchsbedingungen waren so gewählt, daß für achsennahe Bilder die Höhenunschärfe kleiner als $0,09 \mathrm{~mm}$ und die fokale Randunschärfe kleiner als $0,2 \mathrm{~mm}$ waren.

Das Problem der Deformation der Elektroden unter dem Einfluß des elektrischen Überschlags verdient besondere Beachtung. Man kann nämlich gegen die Auswertung der Funkenschallbilder folgenden Einwand erheben (Abb. 6): Unter dem Druck der Entladung könnten die Elektroden $e_{1}$ und $e_{2}$ örtlich zurückweichen, die aus dem Entladungsraum verdrängte Flüssigkeit den Verdichtungsraum D so erfüllen, daß vorwiegend eine Vermehrung der Schichtdicke und dadurch eine Erhöhung der Röntgenabsorption die Folge wäre. Da die Deformation der Elektroden schneller läuft als die Ausbreitung von $\mathrm{D}$, müßten oberhalb und unterhalb der Flüssigkeitsplatte Fl durch Kavitation Hohlräume $\mathrm{H}$ entstehen. Entgegen dieser Möglichkeit ergeben die Experimente, daß das Funkenschallbild kein unter hydrostatischem Druck stehendes Gebilde darstellt, also keinen allseitig gleich wirkenden Druck ausübt. Nur der Entladungsraum $\mathrm{E}$ dürfte für sich allein weitgehend dieser Bedingung genügen. Er versetzt durch seine radiale 
Ausbreitung die im Gegensatz zu den festen Elektroden stark kompressible Flüssigkeit im Verdichtungsraum D in einen Spannungszustand. Der Druck, unter dem der Verdichtungsraum D steht, weist keine, oder jedenfalls nur sehr schwache Komponenten in Richtung auf die Elektroden auf. Der Verdichtungsraum „gleitet" zwischen den Elektroden in Richtung des Spannungsvektors. Die nähere Untersuchung der Ausbildung und Gestalt komplizierterer Schallfiguren (z. B. mit Kopfwellen) spricht gegen eine Elektiodendeformation. Wie wir noch sehen werden, gehen bei kleinen Elektrodenabständen die Entladungen nicht nur durch einen Hauptkanal, sondern durch viele kleine Kanäle über eine größere Elektrodenfläche vor sich. Das wäre in unmittelbarer Nähe des Verdichtungsraumes der Hauptentladung nicht möglich, wenn durch Hohlraumbildung über der Flüssigkeit die Stromleitung zwischen den Elektroden unterbrochen wäre (vgl. Abb. 12).

Eine Deformation der Elektroden mïßte sich sichtbar machen lassen, wenn man eine Röntgendurchleuchtung senkrecht zur Funkenbahn vornimmt und extreme, eine Deformation begünstigende Bedingungen wählt. In Abb. 7 dienen als Elektroden Aluminiumkappen von $0,65 \mathrm{~mm}$ Stärke und einem Krümmungsradius von $43 \mathrm{~mm}$ am Orte ihres geringsten Abstandes. Als Flüssigkeit wurde Benzin genommen, da eine Trichloräthylenschicht von $70 \mathrm{~mm}$ Tiefe hier keine Röntgenstrahlung mehr durchgelassen hätte. An die $1 \mathrm{~mm}$ voneinander entfernten Elektroden wurde eine Spannung von $80 \mathrm{kV}$ gelegt. Es ergibt sich, daß während des Ablaufs der Funkenschallwelle zwar eine (bleibende) Verbeulung der Elektroden eintritt, diese sich aber praktisch auf den Durchmesser des Entladungsraumes beschränkt. Das Volumen des Verdichtungsraumes wird nur um etwa $8 \%$ vergrößert. Auf die Berechnung der mittleren Dichte des Verdichtungsraumes hat diese Korrektur nur einen geringen Einfluß. Der gleiche Versuch, ausgeführt an $2 \mathrm{~mm}$ starken Aluminiumelektroden, ergab weder beim Elektrodenabstand $1 \mathrm{~mm}$, noch bei $0,5 \mathrm{~mm}$ einen meßbaren Einflul.

\section{Meßergebnisse}

1. Die Ausdehnung der Funkenschallbilder

Die Ausdehnung der Funkenschallbilder hängt stark vom jeweiligen Elektrodenabstand bzw. von der örtlichen Dicke der Flüssigkeitsschicht ab. Abb. 8 gibt eine Darstellung der Abhängigkeit des Radius $r_{\mathrm{a}}$ des Verdichtungsraumes und des Radius $r_{\mathrm{i}}$ des Entladungsraumes (vgl. Abb. 1) vom Elektrodenabstand $d$ im Zeitmoment $t=23,5 \cdot 10^{-6} \mathrm{sec}$ nach Beginn des Durchschlags wieder. Versuchsflüssigkeit ist Trichloräthylen. Die elektrische Schaltung ist die gleiche wie in Abb. 5 (bzw. Abb. 3 in S. u. T.); Versuchsgefäß nach Abb. 3. Oberhalb $d=3 \mathrm{~mm}$ wird die Ausbreitung der Funkenschallwellen vom Elektrodenabstand un- abhängig, unterhalb $d=1 \mathrm{~mm}$ nimmt sie sehr schnell ab. Bei gegebenem Elektrodenmaterial wird die Absorption der Röntgenstrahlen mit abnehmender Flüssigkeitsschicht sehr gering. so daß der Strahlenkontrast recht schwach wird. Charakteristisch ist, daß die Ausdehnungskurven

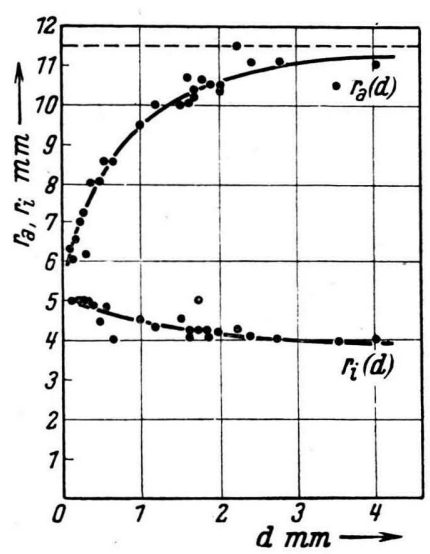

Abb. 8. Die Radien $r_{\text {a }}$ und $r_{\text {i }}$ der Funkenschallbilder als Funktion des Elektrodenabstandes $d$ an der Durchschlagsstelle nach $23,5 \cdot 10^{-6}$ sec. Trichloräthylen, $50-60 \mathrm{kV}, 850 \mathrm{Amp}$.

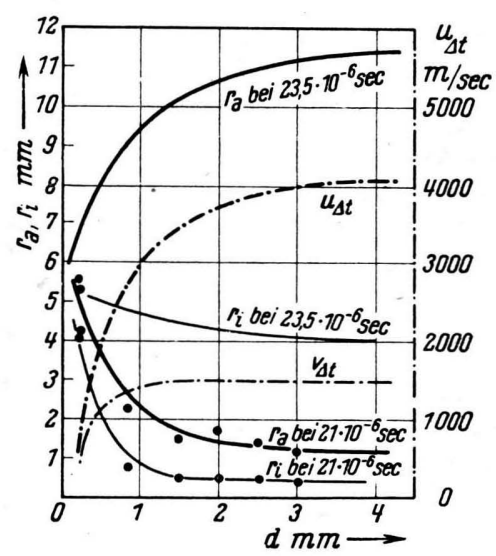

Abl. 9. Vergleich der Radien $r_{\mathrm{a}}$ und $r_{\mathrm{i}}$ bei $t=23,5 \cdot 10^{-6} \mathrm{sec}$ mit denen bei $t=21 \cdot 10^{-6} \mathrm{sec}$ als Funktion von $d$. Trichloräthylen.

für Entladungs- und Verdichtungsraum für $d=0$ offenbar gegen einen gemeinsamen Punkt konvergieren. Exakt ist dieser Punkt nicht zu verwirklichen, schon weil die Bedingung, daß bei gegebenen Werten von Strom und Spannung nur ein Funkenkanal entsteht, nicht mehr zu erfüllen ist. Das Ansteigen des Radius des Entladungsraumes mit abnehmendem Elektrodenabstand ist verständlich, denn es wird ja die Dichte der vom Kondensator gelieferten Entladungsenergie höher. 
Dagegen dürften die Ursachen des starken Rückganges des Verdichtungsraumes wesentlich schwieriger zu erfassen sein. Vermutlich spielen Wärmeleitung und innere Reibung, insbesondere Wandreibung, eine ausschlaggebende Rolle, wenn nicht noch andere, mit der starken Dispersion der Schallgeschwindigkeit auf diesen kurzen Wegstrecken zusammenhängende Gründe maßgeblich sind.

Für die aus Abb. 8 ersichtlichen, bisweilen nicht unerheblichen Schwankungen in der Ausdehnung der Funkenschallbilder kommen zwei Ursachen in Frage. Die Verzögerungszeit $t$, gemessen mit den Schaltungen nach Abb. 4 und 5, ist mit einer Unsicherheit behaftet, die ihre Gründe in den oben erwähnten Schaltelementen, z. B. Funkenstrecken mit ihren statistischen Eigenschaften, hat. Und so wie die Lage des Durchschlagspunktes wohl infolge von Verunreinigungen schwankt, werden sich aus dem gleichen Grunde bei der hohen elektrischen Feldstärke zwischen den Elektroden die Entladungen nicht ausschließlich an einer Stelle abspielen, sondern über größere Flächen verteilen, ohne infolge ihrer geringen Dichtewirkungen in unseren Bildern sichtbar zu werden.

In Abb. 9 sind die beiden Kurven der Abb. 8 noch einmal gezeichnet worden. Ferner sind neu eingetragen die Ausdehnungskurven von Verdichtungs- und Entladungsraum in dem Zeitmoment $t=21 \cdot 10^{-6}$ sec nach dem Durchschlag. Es konnten hierbei nur wenige Meßpunkte aufgenommen werden, sie lassen aber das Wesentliche erkennen. Unterhalb eines Elektrodenabstandes $d=1 \mathrm{~mm}$ verändert sich das Bild beträchtlich und nähert sich dem der Kurven für spätere Zeitmomente. Es macht sich die schon erwähnte Tatsache geltend, daß die Energiedichte bei einem Entladungsraum sehr geringer Schichtdicke groß wird. Demgemäß muß auch die Schallabstrahlung vom Entladungsraum wesentlich früher als bei der vorgenannten Abb. 8 eingesetzt haben.

Wie schon erwähnt wurde, ist bei sehr kleinem Elektronenabstand der Bildkontrast in Trichloräthylen gering. Man kann ihn beträchtlich erhöhen, wenn man als Versuchsflüssigkeit einen Stoff nimmt, der sich durch ein hohes Atomgewicht einer seiner Komponenten auszeichnet, also einen gegen Aluminium großen Schwächungskoeffizienten für Röntgenstrahlen besitzt. Als Belege für Art und Unterschied solcher Kurven für $t=21 \cdot 10^{-6} \mathrm{sec}$ und $t=30 \cdot 10^{-6} \mathrm{sec}$ bei einem
Elektrodenabstand $d=0,2 \mathrm{~mm}$ dienen die Aufnahmen Abb. 11 und 12 an Brombenzol $\mathrm{C}_{6} \mathrm{H}_{5} \mathrm{Br}^{3}$.

Vermindert man die Stromstärken im Entladungskreis durch Einschaltung von Ohmschen Widerständen, so verändern sich die Proportionen der Funkenschallbilder nach Abb. 10. Der Radius $r_{\mathrm{i}}$ des Entladungsraumes $\mathrm{E}$ nimmt linear mit der Stromstärke $a b$ und konvergiert erwartungsgemäß gegen 0 . Der Radius $r_{\mathrm{a}}$ des Verdichtungsraumes D muß dagegen einem endlichen Wert zustreben, da auch für die schwächsten Funken die Schallgeschwindigkeit nicht unter ihren Normalwert $1050 \mathrm{~m} / \mathrm{sec}$ fallen kann. Wie aus der Abb. 10 hervorgeht, konvergiert das Verhältnis $r_{\mathrm{i}}: r_{\mathrm{a}}$ gegen 0 , die nach Gl. (1) unten davon abhängige

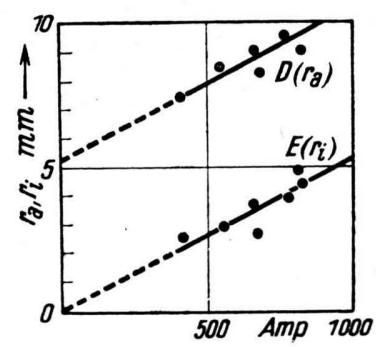

Abb. 10. Verdichtungsraum D $\left(r_{\mathbf{a}}\right)$ und Entladungsraum E $\left(r_{\mathrm{i}}\right)$ als Funktion der max. Anfangsstromstärke der Entladung. $t=24 \cdot 10^{-6} \mathrm{sec}, d \approx 1,7 \mathrm{~mm}$.

Verdichtung $\varrho_{u}$ : $\varrho$ des Verdichtungsraumes noch schneller; d.h. für $300 \mathrm{Amp}$. läßt sich eine nach Gl. (1) berechnete Verdichtung des Trichloräthylens um $+5 \%$ noch gerade nachweisen, für $200 \mathrm{Amp}$. die entsprechende Verdichtung um $+0,3 \%$ nicht mehr.

\section{Die Schallgeschwindigkeit}

In zwei Punkten weicht die Schallgeschwindigkeit von Funkenschallwellen vom normalen Verhalten gewöhnlicher Schallwellen beträchtlich ab, einmal in der starken Abhängigkeit von der Schichtdicke des Mediums, in dem sie laufen, zum anderen in ihrer räumlichen und zeitlichen Veränderlichkeit. Wenn man in Abb. 9 die Differenzen der Ordinaten der beiden $r_{\mathrm{a}}$-Kurven durch den Zeitunterschied, für den sie aufgenommen worden sind, dividiert, so erhält man als mittlere Schallgeschwindigkeit $u_{\Delta \iota}$. Die obere strichpunktierte

3 Sämtliche Aufnahmen sind Positivkopien der Original-Negative; d. h. den Stellen größerer Helligkeit entspricht die geringere Röntgenstrahlenabsorption und umgekehrt. 
Kurve der Abb. 9 stellt $u_{\Delta t}$ als Funktion des Elektrodenabstandes $d$ dar. Sie ist für größere Abstände 3-4-mal so groß wie die gewöhnliche, als molekulare Konstante geltende Schallgeschwindigkeit von $1050 \mathrm{~m} / \mathrm{sec}$ bei $20^{\circ} \mathrm{C}$ in Trichloräthylen und sinkt für Flüssigkeitsschichten von einigen Zehntelmillimetern Dicke rasch unter diesen Normalwert $a b$.

Die untere strichpunktierte Kurve der Abb. 9 stellt die mittlere Ausdehnungsgeschwindigkeit $v_{\lrcorner t}$ des Entladungsraumes im gleichen Zeitabschnitt dar. Sie ist viel weniger von der Begrenzung des Mediums abhängig und wird für kleine Abstände $d$ unsicher, breitet sich aber immerhin in diesem Zeitabschnitt zwischen 20 und 24 Mikrosekunden auch mit Überschallgeschwindigkeit aus.

Bei einem Vergleich der Schallgeschwindigkeiten bei größeren Elektrodenabständen mit dem früher in S. u. T. angegebenen Wert $1700 \mathrm{~m} / \mathrm{sec}$ fällt auf, daß jene etwa doppelt so groß sind wie dieser. Dabei ist aber zu beachten, daß die frühere Bestimmung mit einer kleineren Entladungsstromstärke und bei viel größeren $\Delta t$-Werten ( $\Delta t$ etwa $\left.7 \cdot 10^{-6} \mathrm{sec}\right)$ vorgenommen worden ist, ohne die starke Dispersion der Schallgeschwindigkeit mit der Zeit zu kennen. Bei der Schallgeschwindigkeitskurve der Abb. 9 ist das Zeitintervall mit $2,5 \cdot 10^{-6}$ sec schon wesentlich kleiner, aber die ermittelte Schallgeschwindigkeit gibt auch hier nur einen Mittelwert in diesem Zeitintervall an.

Dieser Befund läßt vermuten, daß bei noch kürzerer Zeitspanne noch größere wahre Schallgeschwindigkeiten auftreten, und er wird dadurch gestützt, daß es mit den augenblicklichen Hilfsmitteln nur selten gelingt, unter den gegebenen Bedingungen für $21 \cdot 10^{-6} \mathrm{sec}<t<22 \cdot 10^{-6} \mathrm{sec}$ Funkenschallbilder vom äußeren Radius $2 \mathrm{~mm}$ $<r_{\mathrm{a}}<8 \mathrm{~mm}$ und vom inneren Radius $1 \mathrm{~mm}$ $<r_{\mathrm{i}}<3 \mathrm{~mm}$ zu erhalten. Das ist nur so zu erklären, daß nach der deutlichen Differenzierung des Funkenschallbildes im Entladungs- und Verdichtungsraum eine mit sehr hoher Überschallgeschwindigkeit in $\Delta t \approx 1 \cdot 10^{-6}$ sec ablaufende explosionsartige Aufweitung stattfindet. Es ist daher auch keineswegs einfäch, in der Kurvenschar, zu der die beiden Kurven $r_{\mathrm{a}}(t)$ in Abb. 9 gehören, diejenigen zu ermitteln, die der unteren naheliegen bzw. ungefähr horizontal verlaufen.

Abb. 13 zeigt für Elektrodenabstände zwischen 1 und $2 \mathrm{~mm}$ die Ausdehnungskurven $r_{\mathrm{a}}(t)$ der
Front des Verdichtungsraumes und $r_{\mathrm{i}}(t)$ des Entladungsraumes. Die strichpunktierte Kurve ist durch Differentiation erhalten und stellt die wahre Schallgeschwindigkeit $u$ (identisch mit Ausdehnungsgeschwindigkeit der Front des Verdichtungsraumes) dar. Die Änderung der Schallgeschwindigkeit mit der Zeit ist also beträchtlich. Man kann 5 Zeitabschnitte unterscheiden:

1. $t_{1} \approx 10^{-7} \mathrm{sec}, r_{\mathrm{a}} \equiv r_{\mathrm{i}} \leqq 0,5 \mathrm{~mm}$.

2. $t_{2} \equiv \tau \approx 20 \cdot 10^{-6}$ sec. Die Differenzierung $r_{\mathrm{a}}>r_{\mathrm{i}}$ beginnt langsam. Ausbildung des Verdichtungsraumes als Dichtering. Verwaschene Ưbergänge.

3. $t_{3} \approx 1 \cdot 10^{-6}$ sec. Explosionsartige Aufweitung des Funkenschallbildes. $r_{\mathrm{a}}>r_{\mathrm{i}}$. Die Schallgeschwindigkeit erreicht abnorm hohe Werte $u \rightarrow 10000 \mathrm{~m} / \mathrm{sec}$. Abb. 14 und 15 .

4. $t_{4} \approx 8 \cdot 10^{-6}$ sec. Allmählich wird $r_{\mathrm{a}} \gg r_{\mathrm{i}}$. Die Überschallgeschwindigkeit sinkt zunächst schnell, dann langsam ab, behält aber ihren über dem Normalen liegenden Wert. Der Verdichtungsraum ist einigermaßen gleichmäßig von Schallenergie erfüllt und zeigt seine charakteristische Ausprägung. Abb. 16 und 17. 5. $t_{4}<t_{5}<\infty$. Die Schallgeschwindigkeit ist auf ihren Normalwert abgesunken. Der Entladungsraum dehnt sich kaum mehr aus und wird sich schließlich zusammenziehen und verschwinden.

Die auf der Abszisse noch vermerkte Zeitspanne $t_{i}$ gibt an, wann bei diesen Versuchen die oszillatorische Entladung von $600 \mathrm{kHz}$ von ihrem anfänglichen Maximalwert von etwa $850 \mathrm{Amp}$. auf Null abgesunken ist.

Da die Genauigkeit der Zeitmessung aus den früher erwähnten Gründen etwa $\pm 1 \cdot 10^{-6}$ sec und damit von der Größe $t_{3}$ ist, kann das angegebene Maximum der Schallgeschwindigkeit nur als Anhaltspunkt für den ungefähren Wert angesehen werden. Es ist nicht ausgeschlossen, daß bei vielmals stromstärkeren Funkenentladungen Geschwindigkeitsmaxima von $100 \mathrm{~km} / \mathrm{sec}$ auftreten können, für deren scharfe Erfassung die Kürze unserer Röntgenblitze bestimmt nicht mehr ausreicht. Sicher ist, daß der Verdichtungsraum im Zeitabschnitt $t_{3}$ eine Unschärfe aufweist, bei der nicht mit Sicherheit entschieden werden kann, ob sie lediglich auf die noch zu geringe Röntgenstrahlenabsorption oder hauptsächlich auf die genannten Gründe zurückzuführen ist; $\mathrm{Abb} .15$ gibt ein Beispiel. 
Die Bestimmung des Funkenschallbildes im Zeitraum $t_{1}$ wurde mit der Versuchsanordnung nach Abb. 2 durchgeführt. Da sich die photographische Aufnahme zur Reproduktion wenig eignet, ist auf eine bildliche Wiedergabe dieses Stadiums verzichtet worden. Eine nähere Diskussion der Stromstärke des Funkens im Augenblick der Aufnahme ergab einen Wert um 1500 Amp. Es wird bei einem Elektrodenabstand von 1,5 mm an der Durchschlagsstelle ein verwaschener Kanal schwommenen Charakter der Erscheinung nichts geändert.

Im Zeitraum $t_{4}$ sinkt die Geschwindigkeit der Schallwellenfront sehr schnell ab, und die aus dem Entladungsraum nacheilenden schnelleren Schallwellen erfüllen den Verdichtungsraum so, daß er auf den ersten Blick gleichmäßig dunkel erscheint. Die nacheilenden Wellen bewirken gleichzeitig die Versteilung der Front. Die geringere Ausbreitungsgeschwindigkeit gestattet eine

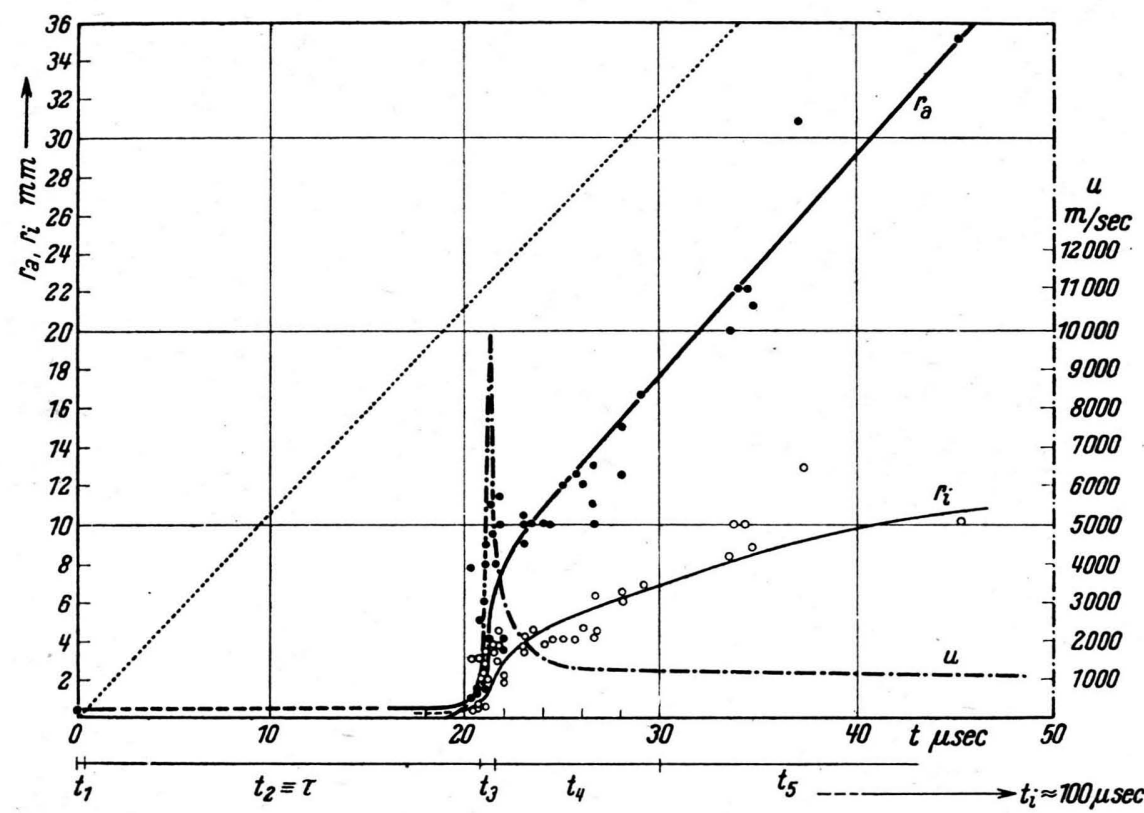

Abb. 13. Die Radien $r_{a}$ und $r_{i}$ (Verdichtungsraum und

Entladungsraum) als Funktion der Zeit $t$ bei einem Elektrodenabstand $d$ zwischen 1,5 und 2 mm. Strichpunktiert die Schallgeschwindigkeit (der Frontwelle des Verdichtungsraumes) als Funktion der Zeit. Die durch den Koordinaten-Nullpunkt gehende punktierte Gerade deutet an, wie bei einer normalen Schallgeschwindigkeit in Trichloräthylen von $1050 \mathrm{~m} / \mathrm{sec}$ bei sofortiger Emission der Verlauf von $r_{\mathrm{a}}$ sein würde.

von etwa $0,75 \mathrm{~mm}$ Breite schwach sichtbar. Da wir es im vorliegenden Falle mit dem Durchschlag eines nichtoszillierenden Funkens zu tun haben, findet sich sehr viel Energie in diesem Kanal konzentriert. Offenbar liegt noch keine Trennung von Flüssigkeits- und Gasphase vor, und es existiert daher auch noch nicht der später den Entladungsraum so charakteristisch umgebende ringförmige Verdichtungsraum. In der Zeit von $10^{-7} \mathrm{sec}$ nach Durchschlag kann man noch nicht von einer Emission von Funkenschallwellen sprechen. Wird der Abstand der Elektroden verkleinert, so daß die Länge des Durchschlagraumes $0,5 \mathrm{~mm}$ beträgt, so wird sein Durchmesser zwar auf etwa $1 \mathrm{~mm}$ vergrößert, aber an dem ver- schärfere Erfassung durch die Röntgenblitze. Die $r_{\mathrm{a}}(t)$-Kurve muß asymptotisch in eine Gerade mit der Steilheit $d r_{\mathrm{a}} / d t=u_{\text {normal }}=1050 \mathrm{~m} / \mathrm{sec}$ übergehen. Abb. 15 und 16 geben charakteristische Bilder dieses Zeitabschnittes.

Die Kurven der Abb. 13 erfahren unter anderen Versuchsbedingungen eine Verschiebung, ohne ihren Charakter einzubüßen. In dünneren Flüssigkeitsschichten verlaufen die Schallwellen langsamer, die Ausbreitungsgeschwindigkeit des Entladungsraumes wird ausschlaggebender und die Verzugszeit $\tau$ wird wesentlich kleiner. Der charakteristische Knick der $r_{\mathrm{a}}(t)$ - und $r_{\mathrm{i}}(t)$-Kurven wird flacher.

Die Ausdehnungsgeschwindigkeit des zentralen 
Entladungsraumes, die einen ähnlichen Verlauf wie die $u$-Kurve in Abb. 13 aufweist und ein Maximum von etwa $4000 \mathrm{~m} / \mathrm{sec}$ durchläuft, ist durch die kinetische Energie der Bestandteile des Plasmas bestimmt und läuft nur anfänglich der des Verdichtungsraumes parallel. Sie verlangsamt sich bald; die Ausdehnung $r_{i}$ des Entladungsraumes dürfte nach etwa $50 \cdot 10^{-6}$ sec ihr Maximum finden.

3. Die Erzeugung hoher Dichten und Drucke

Wie aus Abb. 8 hervorgeht, nimmt in der Zeitphase $t_{4}$ mit abnehmendem Elektrodenabstand $d$ der Unterschied in den Radien von Verdichtungsraum und Entladungsraum ab. Darauf beruht die Möglichkeit, Materie kurzzeitig sehr stark zu komprimieren. In der für eine Flüssigkeitsplatte in S. u.T. abgeleiteten Formel für die mittlere Dichte $\varrho_{u}$ des Verdichtungsraumes

$$
\varrho_{u}=\varrho \frac{1}{1-\left(r_{\mathrm{i}} / r_{\mathrm{a}}\right)^{2}}
$$

strebt $r_{\mathrm{i}} \rightarrow r_{\mathrm{a}}$ und mithin $\varrho_{u} \rightarrow \infty$. Aber schon die Erzielung einer dreifachen Dichte bereitet Schwierigkeiten. Zu ihren Ursachen gehört die schon erwähnte Tatsache, daß bei engen Elektrodenabständen der Funkenüberschlag nicht mehr an einer einzelnen Stelle erfolgt, sondern über einen größeren Elektrodenbereich in zahllosen, mehr oder minder feinen Kanälen. Zwar bildet sich im allgemeinen ein einzelner Kanal zum Funkenschallbild besonders stark aus, doch kann die ihm zugeführte Energie durch die Nebenkanäle bedeutend geschwächt sein.

In Tab. 1 sind einige organische Flüssigkeiten zusammengestellt worden, an denen Versuche zur Erzielung hoher Dichten gemacht wurden (Apparatur nach Abb.3). In der Tabelle sind die Normaldichten o bei $20^{\circ} \mathrm{C}$, die Dichtesteigerungen $\varrho_{u}: \mathrm{g}$ und die maximal erzielten Dichten $\mathrm{o}_{n}$ verzeichnet. Die vorletzte Spalte enthält die Angabe des Elektrodenabstandes $d$ an der Stelle der Dichtebestimmung. Die Dichten $\varrho_{u}$ können naturgemäß nicht mit Temperaturangaben versehen werden, da diese hier auf experimentellem Wege nicht zu ermitteln sind.

Aus den Abb. 11 und 12 ist deutlich zu ersehen, daß die Entladung sich über verschiedene Kanäle verteilt hat, von denen jeder für sich ein Funkenschallbild im Kleinen erzengt. Dadurch, daß bei

\begin{tabular}{|l|l|l|l|l|l|}
\hline \multicolumn{1}{|c|}{ Flüssigkeit } & Formel & $\underset{\mathrm{g} / \mathrm{cm}^{3}}{\varrho}$ & $\varrho_{n}: \varrho$ & $\begin{array}{c}\varrho_{u} \\
\mathrm{~g} / \mathrm{cm}^{3}\end{array}$ & $\begin{array}{c}d \\
\mathrm{~mm}\end{array}$ \\
\hline Trichloräthylen & $\mathrm{C}_{2} \mathrm{HCl}_{3}$ & 1,48 & 3,27 & 4,84 & 0,10 \\
a-Chlornaphthalin & $\mathrm{C}_{10} \mathrm{H}_{7} \mathrm{Cl}$ & 1,19 & 2,06 & 2,45 & 0,22 \\
Brombenzol & $\mathrm{C}_{6} \mathrm{H}_{5} \mathrm{Br}$ & 1,50 & 2,55 & 3,82 & 0,22 \\
Todbenzol & $\mathrm{C}_{6} \mathrm{H}_{5} \mathrm{~J}$ & 1,83 & 2,00 & 3,66 & 0,2 \\
Äthylenbromid & $\mathrm{C}_{2} \mathrm{H}_{4} \mathrm{Br}_{2}$ & 2,06 & 2,27 & 4,68 & 0,2 \\
Schwefelkohlenstoff & $\mathrm{CS}_{2}$ & 1,26 & 3,27 & 4,12 & 0,3 \\
\hline
\end{tabular}

Tab. 1. Zusammenstellung hoher Dichten in einigen Flüssigkeiten. Strom etwa $850 \mathrm{~A} ; t=24 \cdot 10^{-6} \mathrm{sec}$, nur bei Brombenzol $t=30 \cdot 10^{-6}$ sec.

der schnelleren Ausbreitung der Hauptentladıng schwächere Nebenentladungen geschluckt werden, entstehen die unregelmäßigen Umrandungen von Entladungs- und Verdichtungsräumen.

Die in den Verdichtungsräumen herrschenden dynamischen Drucke können wohl nur auf Grund des Zusammenhanges mit Schallgeschwindigkeit und Dichte, wie ihn die Theorie der Stoßwellen liefert, berechnet werden ${ }^{4}$. Für die Ausbreitungsgeschwindigkeit $u$ einer schnellen Schallwelle gilt dann

$$
u^{2}=v^{2} \frac{p_{u}-p}{v-v_{u}},
$$

wenn wir unter $p$ den Druck vor und unter $p_{u}$ den Druck hinter der Wellenfront, unter $v$ das spezif. Volumen vor und unter $v_{u}$ hinter der Wellenfront verstehen. Etwas anders geschrieben lautet diese Gleichung:

$$
u^{2}=\frac{\varrho_{u}}{\varrho} \frac{\Delta \mu}{\Delta \underline{\Delta}},
$$

wenn wir unter $\varrho_{u}$ und $\varrho$ die Dichten hinter und vor der Wellenfront verstehen und den Druckunterschied mit $\Delta_{p}$ und den Dichteunterschied $\left(\varrho_{u}-\varrho\right)$ mit $\Delta \mathrm{Q}$ bezeichnen. Für sehr kleine Dichteänderungen geht diese Formel in die bekannte Beziehung für die normale Schallgeschwindigkei $u^{2}=d p / d \varrho$ über. Aus (3) ergibt sich für den (gegen Atmosphärendruck gemessenen) Druck I" der Wert

$$
P_{u}=\Delta_{p}=u^{?} \stackrel{Q}{Q_{u}} J_{Q} .
$$

Seine genaue Bestimmung in jedem Punkt des Verdichtungsraumes macht eine örtliche Dichtemessung von $\varrho_{u}$ erforderlich. Diese setzt eine röntgenographische Schwärzungsmessung voraus. die aber infolge der unbekannten Spektralverteilung der Röntgenblitzstrahlung einstweilen zu-

$$
4 \text { S. a. R. Becker, Z. Physik 8, 321-362 [1922]. }
$$


rückgestellt werden muß. Für den oben beschriebenen Zeitabschnitt $t_{4}$ der Entwicklung der Funkenschallbilder aber können wir auf die geometrische Formel (1) zurückgreifen. Sie gilt für Bilder des Typus Abb. 16, 17, 18.

Durch ihren Einsatz in Gl. (4) erhalten wir daher

$$
p_{u}=u^{2} \varrho\left(r_{\mathrm{i}} / r_{\mathrm{a}}\right)^{2} \text {. }
$$

Wenn man nun auf der Grundlage der Kurven der Abb. 13 die Funktion $p_{u}=f(t)$ nach Gl. (5) zeichnet, so erhält man Abb.19. Um die auf die Kurven der Abb. 13 zurückgehenden Fehlermöglichkeiten zum Ausdruck zu bringen, ist für den Zeitabschnitt $t_{4}$ die Zeitkurve des dynamischen

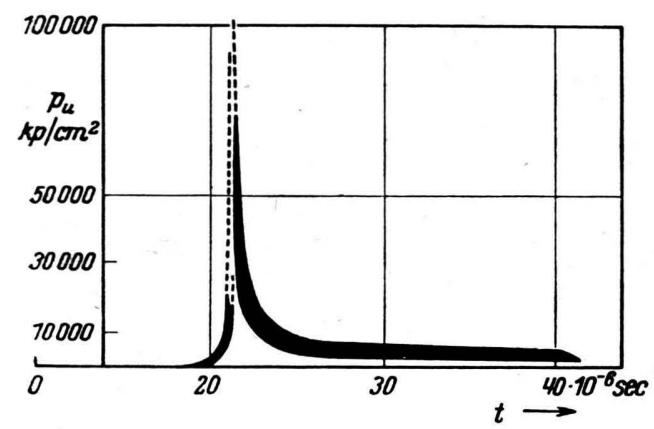

Abb. 19. Zeitverlauf des dynamischen Druckes hinter der Schallwellenfront auf Grund der Kurven der Abb. 13; für den Zeitabschnitt $t_{3}$, wo Gl. (1) nicht angewandt werden kann, nur punktiert angedeutet.

Druckes als breites Band und für den Zeitabschnitt $t_{3}$ nur gestrichelt gezeichnet worden. Sie zeigt, daß der Druck äußerst kurzzeitig sehr große Werte annehmen kann, aber in dem bislang gut zugänglichen Bereich des Zeitabschnittes $t_{4}$ Werte zwischen 10000 und $50000 \mathrm{kp} / \mathrm{cm}^{2}$ besitzt. So errechnet sich beispielsweise für den Verdichtungsraum in Abb.17 ein dynamischer Druck, der zwischen 3000 und $6000 \mathrm{kp} / \mathrm{cm}^{2}$ liegt, für Abb. 16 ein solcher zwischen 8000 und $14000 \mathrm{kp} / \mathrm{cm}^{2}$, für Abb. 18 ein solcher zwischen 10000 und $20000 \mathrm{kp} / \mathrm{cm}^{2}$ und für Abb. 12 zwischen 8000 und $16000 \mathrm{kp} / \mathrm{cm}^{2}$. Unmittelbar hinter der Wellenfront der Funkenschallwellen tritt nach der Theorie eine Strömungsgeschwindigkeit $w$ der Flüssigkeit auf, die gegeben ist durch

$$
u=\left(v-v_{u}\right) \sqrt{\frac{p_{u}-p}{r-v}} \text { bzw. } u^{2}=\frac{\Delta p \Delta \varrho}{\varrho \varrho_{u}} .
$$

Ersetzen wir $\Delta p$ durch Gl. (4), so wird

$$
w=u\left(1-Q / Q_{u}\right) \text {. }
$$

In dem speziellen Fall der Anwendbarkeit der Gl. (1) erhalten wird dann

$$
u=u\left(r_{\mathrm{i}} / r_{\mathrm{a}}\right)^{2} \text {. }
$$

Diese Strömung, die beim Auftreffen der Wellen auf Hindernisse eine Rolle spielt, nimmt in den vorliegenden Fällen Werte zwischen 250 und $500 \mathrm{~m} / \mathrm{sec}$ an.

Das Auftreten von Nebenentladungen macht es unwahrscheinlich, daß eine Steigerung der Entladungsstromstärke uns gestatten wird, unter den vorliegenden Bedingungen wesentlich größere Dichten, als die Tabelle verzeichnet, zu erzeugen. Man kann aber versuchen, zwei Funkenschallwellen gegeneinander laufen zu lassen, um durch ihren Zusammenprall höhere Dichten und Drucke zu erreichen. Unter einer größeren Anzahl von Funkendurchschlägen kommt der Fall vor, daß sich die Entladung in zwei fast gleichwertigen, nebeneinander liegenden Kanälen abspielt, wie Abb. 20 zeigt. Sie gilt für einen Elektrodenabstand $d=0,4 \mathrm{~mm}$ im Zeitmoment $t=24 \cdot 10^{-6} \mathrm{sec}$ nach Durchschlag. Eine nähere Untersuchung zeigt nun aber, daß der nach dem Zusammenprall sich bildende Flüssigkeitssteg zwar unter einem hohen Druck, aber infolge seiner geringen Breite noch mehr unter der ungeheuren Temperatureinwirkung der beiden Entladungsräume sowie seiner Eigentemperatur stehen muß, so daß er in die Entladungsräume hinein verdampft und die in ihm fehlende Materie sich kondensiert im gesamten äußeren Verdichtungsraum wiederfindet und dessen Dichte zusätzlich erhöht. Für diese Verdampfung spricht auch das wellige zerfaserte Aussehen des Steges im Original.

\section{Funkenschallwellen an der Grenze f ester Körper}

Es ist bis jetzt noch nicht gelungen, eine durch Funkenüberschlag in einem festen Stoff entstandene oder aus einer Flüssigkeit in ihn eindringende Schallwelle mit Hilfe von Röntgenlichtblitzen nachzuweisen. Versuche darüber wurden ausgeführt an Igelit, Glas, Sinterkorund und Plexiglas. Der Grund für das Ausbleiben einer röntgenographisch sichtbaren Wirkung muß darin gesucht werden, daß die Kompressibilität dieser festen Stoffe zu klein ist, um eine für den Nachweis ausreichende Dichteänderung zu gestatten. Im übrigen erfolgt beim Funkendurchschlag durch eine feste Platte die Schallemission wesentlich 
früher als bei einer Flüssigkeit, längstens einige Mikrosekunden nach dem Durchschlag. Läßt man z. B. den Funkendurchschlag durch eine Glasplatte, die in einer Flüssigkeit liegt, gehen und beobachtet $24 \cdot 10^{-6}$ sec später, so erhält man neben dem bekannten Bild einen Stern von Bruchlinien, die von der Durchschlagsstelle ausgehen. In diesen hohlen Bruchlinien ist die Röntgenstrahlenabsorption gering. Da sie nach den Untersuchungen von $\mathrm{Sch}$ a r d i ${ }^{5}$ später als die Longitudinalund Transversal-Wellen auftreten und eine Geschwindigkeit von etwa $1500 \mathrm{~m} / \mathrm{sec}$ besitzen, muß also die Schallwellenemission im festen Körper schon wesentlich früher als in der Flüssigkeit eingesetzt haben.

Läßt man eine Funkenschallwelle gegen eine feste Platte anlaufen, so wird man erwarten, diesseits der Platte die reflektierte, jenseits der Platte die durchgelassene Welle $\mathrm{zu}$ sehen. Und ist die Schallgeschwindigkeit im festen Stoff größer als in der Flüssigkeit, so ist mit dem Auftreten von Kopfwellen zu rechnen. Die Röntgenaufnahmen aber ergeben ein Bild, welches einmal durch die Eigenart des Funkenschalls in seinem Emissionsgebiet und zum anderen dadurch geprägt ist, daß nur solche Veränderungen wahrgenommen werden, die mit starken Dichteänderungen verknüpft sind. Es ist noch nicht an der-Zeit, alle Details dieses Problems zu behandeln, da die Zusammen-

5 H. Schardin, D. Elle u. W.Struth, Z. techn. Physik 21, 393-400 [1940]. hänge durch die variable Geschwindigkeit der frontalen Schallwelle und die in ihrem Gefolge wirkenden Strömungsvorgänge sehr kompliziert sind.

In der Aufnahme Abb. 21 liegt ein Kamm aus je sechs flachen, 1,6 $\mathrm{mm}$ dicken Igelitstreifen in Trichloräthylen. Die untere Elektrode ist flach, beide Elektroden bestehen aus dünnem Aluminiumblech, mit starkem Pertinax hinterlegt. Die Entfernung Fokus-Film ist $300 \mathrm{~mm}$. Dieser und einer ganzen Reihe ähnlicher, hauptsächlich der Erforschung von Kavitationswirkungen dienenden Aufnahmen, ist die außerordentlich starke örtliche Durchbiegung des Igelits unter dem starken, senkrecht darauf wirkenden Druck gemeinsam. Die mit diesem Druck verbundene Deformation hat schon zu Brüchen, deren Richtung zum Durchschlagszentrum zurückweist, geführt. Die Anregung der Biegewelle in Igelit erfolgt so schnell, daß die Flüssigkeit vom Igelit abreißt und schmale Hohlräume durch Kavitation entstehen. Sie sind neben den Bruchstrecken des Igelits besonders schön an der Grenzlinie Igelit-Trichloräthylen der Abb. $21 \mathrm{zu}$ sehen. In dieser erkennt man an den Verdichtungen der Flüssigkeit deutlich die durch den festen Stoff mit erhöhter Geschwindigkeit gelaufenen Schallwellen. Die mit diesen verbundenen Strömungen führen übrigens später ebenfalls zu Kavitationsbildungen in der Flüssigkeit und an den vom Entladungszentrum abgewandten, von den Schallwellen passierten Oberflächen des Igelits.

\section{NOTIZEN}

\section{Elektrische Eigenschaften von Ruthenium}

$$
\text { Von E. Justi }
$$

Institut für techn. Physik der T. H. Braunschweig

(Z. Naturforschg. 4 a, 472-474 [1949]; eingeg. am 22. August 1949)

Zur Messung der bisher kaum bekannten elektrischen Daten des Rutheniums stellte mir 1943 die Heraeus-Vacuumschmelze Hanau (Dr. Geibel) eine Probe bis dahin noch nicht erreichter Reinheit zur Verfügung. Während z. B. das bisher reinste, von M e i $\&$ ne ${ }^{1}$ untersuchte Präparat derselben Herkunft noch mindestens $4,24 \%$ Verunreinigungen aufwies, enthielt die neue Probe gemäß der Spektralanalyse der Hersteller nur insgesamt $0,01 \% \mathrm{Ca}$ und $\mathrm{Mg}$ sowie kaum merkliche Cu-Spuren. Andere Pt-

1 W. Me i \& ner u. B. Voigt, Ann. Physik 7, $892[1930]$.
Metalle, Au und Ag, waren nicht nachweisbar, so daf das Präparat 99,99\% $\mathrm{Ru}$ enthalten sollte. Das $\mathrm{Ru}-$ Pulver wurde $\mathrm{zu}$ einem parallelepipedischen Stäbchen gepreßt und im $\mathrm{H}_{2}$-Strom bei $1150^{\circ} \mathrm{C}$ vorgesintert, dann im Hochvakuum bei $2200^{\circ} \mathrm{C}$ nachgesintert. Mit einer Länge von $l=4,975 \mathrm{~cm}$, einem über zehn verschiedene Stellen gemittelten Querschnitt von $q=0,221_{56} \cdot 0,219_{70}=0,0486_{8} \mathrm{~cm}^{2}$ sowie einer Masse von $2,798_{5} \mathrm{~g}$ ergibt sich eine Dichte von $s=11,5_{56} \mathrm{~g} / \mathrm{cm}^{3}$, die um $6,1 \%$ hinter der röntgenographisch bestimmten idealen Dichte ${ }^{2} s=12,304 \mathrm{~g} / \mathrm{cm}^{3}$ zurückbleibt und so zeigt, wieweit der Sinterkörper als kompakt anzusprechen ist.

Die mit dem Diesselhorst-Kompensator vorgenommenen Widerstandsmessungen ergaben einen Eispunktswiderstand von $R_{273}=0,0007314_{6} \Omega$, woraus ein spezif. Widerstand $\varrho_{273}=R_{273} q / l=7,15_{71} \cdot 10^{-6} \Omega \mathrm{cm}$

2 L. V. Wh it ney, Physic. Rev. 48, 458 [1935]. 